\title{
Effects of Finerenone Combined with Empagliflozin in a Model of Hypertension-Induced End-Organ Damage
}

\author{
Peter Kolkhofa Elke Hartmann ${ }^{b}$ Alexius Freyberger ${ }^{c}$ Mira Pavkovic ${ }^{d}$ \\ Ilka Mathar ${ }^{\mathrm{a}}$ Peter Sandner ${ }^{\mathrm{a}}$ Karoline Droebner ${ }^{\mathrm{a}}$ Amer Joseph $^{\mathrm{e}}$ Jörg Hüser $^{\mathrm{a}}$ \\ Frank Eitner ${ }^{\mathrm{a}}$ \\ ${ }^{a}$ Cardiovascular Research, Research and Early Development, R\&D Pharmaceuticals, Bayer AG, Wuppertal, Germany; \\ ${ }^{b}$ Research Pathology, Research and Early Development, R\&D Pharmaceuticals, Bayer AG, Wuppertal, Germany; \\ 'Clinical Pathology, Research and Early Development, R\&D Pharmaceuticals, Bayer AG, Wuppertal, Germany; \\ ${ }^{\mathrm{d} B i o m a r k e r ~ R e s e a r c h, ~ R e s e a r c h ~ a n d ~ E a r l y ~ D e v e l o p m e n t, ~ R \& D ~ P h a r m a c e u t i c a l s, ~ B a y e r ~ A G, ~ W u p p e r t a l, ~ G e r m a n y ; ~}{ }^{e} \mathrm{Clinical}$ \\ Development, R\&D Pharmaceuticals, Bayer AG, Berlin, Germany
}

\section{Keywords}

Mineralocorticoid receptor antagonism · Sodium-glucose cotransporter-2 inhibition - Cardiorenal protection .

Anti-fibrotic effects

\begin{abstract}
Introduction: The nonsteroidal mineralocorticoid receptor (MR) antagonist finerenone and sodium-glucose cotransporter-2 (SGLT2) inhibitors have demonstrated clinical benefits in CKD patients with type 2 diabetes. Clinical data analyzing the potential value of a combination therapy are currently limited. We therefore investigated cardiorenal protection of respective mono- and combination therapy in a preclinical model of hypertension-induced end-organ damage. Methods: Cardiovascular (CV) morbidity and mortality were studied in hypertensive, $\mathrm{N}(\omega)$-nitro-L-arginine methyl ester-treated, renin-transgenic (mRen2)27 rats. Rats (10- to 11-week-old females, $n=13-17 /$ group) were treated once daily orally for up to 7 weeks with placebo, finerenone ( 1 and $3 \mathrm{mg} / \mathrm{kg}$ ), empagliflozin ( 3 and $10 \mathrm{mg} / \mathrm{kg}$ ), or a combination of the respective low doses. Key outcome parameters included mortality, proteinuria, plasma creatinine and uric acid, blood pressure, and cardiac and renal histology.
\end{abstract}

karger@karger.com www.karger.com/ajn

Karger"

BOPEN ACCESS
(C) 2021 The Author(s)

Published by S. Karger AG, Basel

This is an Open Access article licensed under the Creative Commons Attribution-NonCommercial-4.0 International License (CC BY-NC) (http://www.karger.com/Services/OpenAccessLicense), applicable to the online version of the article only. Usage and distribution for commercial purposes requires written permission.
Results: Placebo-treated rats demonstrated a 50\% survival rate over the course of 7 weeks. Drug treatment resulted in variable degrees of survival benefit, most prominently in the low-dose combination group with a survival benefit of $93 \%$. Monotherapies of finerenone or empagliflozin dose-dependently reduced proteinuria, while low-dose combination revealed an early, sustained, and over-additive reduction in proteinuria. Empagliflozin induced a strong and dosedependent increase in urinary glucose excretion which was not influenced by finerenone coadministration in the combination arm. Low-dose combination but not respective low-dose monotherapies significantly reduced plasma creatinine and plasma uric acid after 6 weeks. Treatment with finerenone and the low-dose combination significantly decreased systolic blood pressure after 5 weeks. There was a dose-dependent protection from cardiac and kidney fibrosis and vasculopathy with both agents, while low-dose combination therapy was more efficient than the respective monotherapy dosages on most cardiorenal histology parameters. Discussion/Conclusions: Nonsteroidal MR antagonism by finerenone and SGLT2 inhibition by empagliflozin confer CV protection in preclinical hypertension-induced cardiorenal disease. Combination of these 2 independent modes of action at low dosages revealed efficacious reduction in impor-
Correspondence to:

Peter Kolkhof, peter.kolkhof@bayer.com 
tant functional parameters such as proteinuria and blood pressure, plasma markers including creatinine and uric acid, cardiac and renal lesions as determined by histopathology, and mortality indicating a strong potential for combined clinical use in cardiorenal patient populations.

(C) 2021 The Author(s)

Published by S. Karger AG, Basel

\section{Introduction}

There is a high unmet medical need for effective therapies in cardiorenal diseases including heart failure (HF) and CKD. Renin-angiotensin-system blockade, either with angiotensin-converting enzyme inhibitors or angiotensin-receptor blockers, has been included in respective guidelines as antihypertensive cornerstone therapy for $\mathrm{HF}$ with reduced ejection fraction (HFrEF) as well as for CKD with albuminuria $(>300 \mathrm{mg} / \mathrm{g} / \mathrm{d})$ during the last 3 decades $[1,2]$. At least 2 further major pharmacological principles were investigated recently in $\mathrm{HF}$ and $\mathrm{CKD}$ clinical trials beyond blood pressure control by renin-angiotensin-system blockade. First, sodium-glucose cotransporter-2 inhibition (SGLT2i) was introduced as a novel treatment option for metabolic control in type 2 diabetes (T2D). In the EMPA-REG OUTCOME trial, the SGLT2 inhibitor empagliflozin reduced the risk of major adverse cardiovascular (CV) events in patients with T2D at high risk for $\mathrm{CV}$ events [3] and was associated with slower progression of kidney disease and lower rates of clinically relevant renal events in comparison to the placebo [4]. Moreover, in the EMPEROR-Reduced trial, empagliflozin reduced $\mathrm{CV}$ death or HF hospitalization and slowed the progressive decline in kidney function in patients with HFrEF, with and without diabetes [5]. Similarly, the SGLT2 inhibitor dapagliflozin reduced the risk of a composite of a sustained decline in the estimated glomerular filtration rate of at least $50 \%$, end-stage kidney disease, or death from renal or CV causes among patients with CKD, regardless of the presence or absence of diabetes [6]. Thus, SGLT2i provides clinical benefit in HFrEF and CKD independent of its effects on blood glucose.

Second, it is well documented that mineralocorticoid receptor (MR) antagonism has kidney- and cardioprotective effects [7, 8]. MR antagonism provides an important anti-inflammatory/anti-fibrotic mode of action in addition to its well-known natriuretic/anti-edematous pharmacodynamic effects [9]. Conditions of elevated MR expression or upregulation of relevant MR ligands (aldosterone and cortisol), high salt load, increased generation of reactive oxygen species, and ligand-independent activa-

Preclinical Combination Therapy of

Finerenone and Empagliflozin tion by the small GTPase rac-1 may cause a status of the so-called MR overactivation with subsequent expression of pro-inflammatory and pro-fibrotic proteins in different cell types and tissues [8]. Finerenone is a novel, selective, and nonsteroidal MR antagonist, which has demonstrated anti-inflammatory/anti-fibrotic activity in preclinical cardiorenal models, as well as clinical benefits in CKD patients with T2D [8-10]. Most recently, the FIDELIO-DKD phase III study that investigated the efficacy and safety of finerenone in comparison to a placebo in addition to optimized standard of care in 5,734 patients with CKD and T2D met both its primary renal endpoint and its key secondary CV endpoint [11]. Finerenone significantly reduced the risk of the composite $\mathrm{CV}$ outcome which included the time to $\mathrm{CV}$ death, myocardial infarction, stroke, or hospitalization for HF in these patients with no significant interaction between patients with and without CVD [12].

The degree of cardiorenal protection via MR antagonism with finerenone and SGLT2i with empagliflozin in combination is unknown in hypertensive, nondiabetic $\mathrm{CV}$ disease. Therefore, we aimed to investigate cardiorenal protection of respective mono- and combination therapy of finerenone and empagliflozin in a preclinical model of hypertension-induced end-organ damage. We decided to use the transgenic renin mRen 2 rat which develops severe hypertension [13] and hypertension-related pathological lesions in CV tissues including development of progressive left ventricular hypertrophy and glomerulosclerosis [14]. Extensive characterization of transgenic (mRen2)27 rats revealed typical characteristics of hypertensive end-organ damage [15].

\section{Material and Methods}

\section{Animals}

All animal experiments were performed in accordance to the current national legislation (German protection of animal act and the EU directives on the protection of animals used for scientific purposes). All studies were approved by the Regional Regulatory Authority (LANUV NRW in Germany) and by the Institutional Animal Care and Use Committee of Bayer AG. All animals were housed with free access to chow and water and maintained on a light-dark cycle at $22-24^{\circ} \mathrm{C}$.

\section{L-NAME-Treated (mRen2)27 Rats}

Cardiorenal morbidity and mortality was studied in hypertensive and proteinuric renin-transgenic (mRen2)27 rats. The transgenic (mRen2)27 rat is a hypertensive rat line developed by Mullins et al. [13] which overexpresses the mouse Ren-2 gene. Additional administration of the nitrogen monoxide (nitric oxide) synthase inhibitor $\mathrm{N}(\omega)$-nitro-L-arginine methyl ester ( $\mathrm{L}$ - 
NAME) via drinking water induces endothelial dysfunction which increases morbidity and mortality in this model [16]. Unless subjected to life-long antihypertensive therapy, homozygous animals die from secondary complications such as HF and kidney failure or stroke [17]. All animals in the study were initially placed on a diet with the ACE inhibitor captopril (300 mg/ $\mathrm{kg}$ in food) from age 4-5 weeks. A group of 6 (mRen2)27 rats remained on the captopril diet throughout the study and served as a control group, while all other animals were switched to a normal diet from age 8 to 9 weeks for a captopril washout period of 2 weeks. The animals were randomized to treatment groups from age 10 to 11 weeks according to homogenous urinary protein excretion which was not different from captopril-treated control animals determined in a baseline urine collection. LNAME $(20 \mathrm{mg} / \mathrm{L})$ treatment was initiated with drug treatment at randomization, while control animals on the captopril diet received no L-NAME. Survival was defined as the absence of mortality and severe morbidity: all animals were scored on a daily basis by skilled persons, and animals with clear signs of severe morbidity were sacrificed and considered as having reached the mortality endpoint. The study endpoint was predefined with a ca. $50 \%$ mortality rate in the placebo group. At the end of the study, all animals were kept in depth narcotic and bled via aortic puncture and sacrificed by permanent inhaled isoflurane; blood was taken in order to asses plasma NT-proBNP; and hearts and kidneys were weighed and harvested for histopathological and gene expression evaluation.

\section{Compounds and Treatment}

Finerenone was synthesized as previously described [18], and empagliflozin was synthetized at WuXi App Tec, China. Rats (10to 11-week-old female) were treated once daily orally by gavage for up to 7 weeks with vehicle $(10 \% \mathrm{EtOH}, 40 \%$ Solutol, $50 \%$ water; = "placebo" group, $n=17)$ or verum dissolved in vehicle solution: finerenone $1 \mathrm{mg} / \mathrm{kg}(n=14)$, finerenone $3 \mathrm{mg} / \mathrm{kg}(n=13)$, empagliflozin $3 \mathrm{mg} / \mathrm{kg}(n=14)$, empagliflozin $10 \mathrm{mg} / \mathrm{kg}(n=13)$, or a combination of the respective low doses of $1 \mathrm{mg}$ finerenone plus 3 $\mathrm{mg} / \mathrm{kg}$ empagliflozin $(n=14)$. The dosages were chosen based on previous studies in rats $[19,20]$.

\section{Plasma and Urine Collection and Analysis}

Urine samples were collected for $24 \mathrm{~h}$ from rats placed in individual metabolic cages at baseline, week 2 , and week 6 . Plasma samples were collected in week 6 after an overnight fasting period and at study end. Urinary creatinine and glucose, plasma creatinine, glucose, and uric acid were quantified in respective samples using an ADVIA 2400 Analyzer (Siemens Healthcare Diagnostics, Eschborn, Germany) according to the user manual, and total urinary protein was determined using the pyrogallol-molybdate method. Plasma concentrations of rat NT-proBNP were measured with a commercially available sandwich immunoassay kit by electrochemiluminescent detection (Meso Scale Discovery, Gaithersburg, MD, USA) according to the manufacturer's instructions.
Fig. 1. Proportion of survival defined as the absence of mortality and severe morbidity per group over the course of the study.

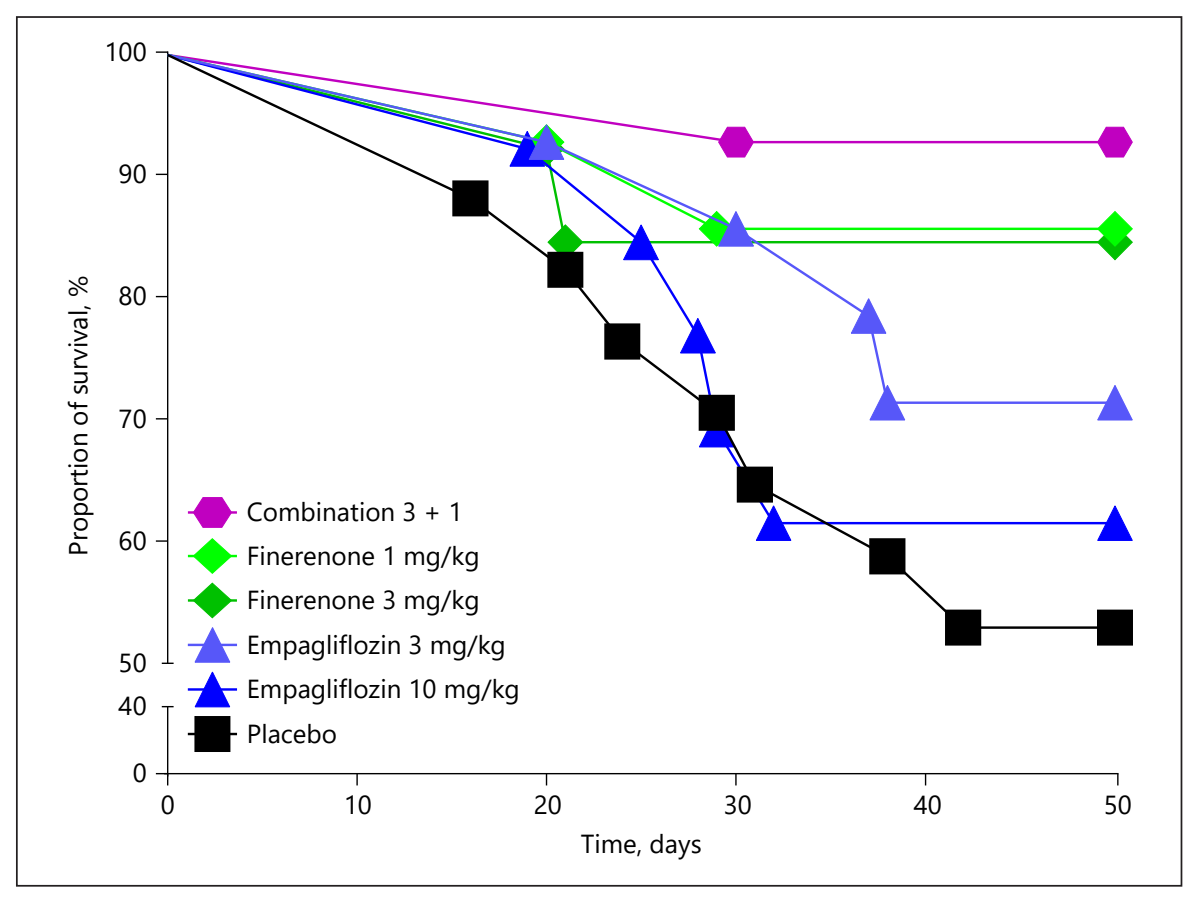

Fig. 2. Urinary and plasma parameters. UPCR determined after 2 (a) and 6 weeks (b), UGCR determined after 2 (c) and 6 weeks (d), plasma creatinine (e), and plasma uric acid (f) determined after 6 weeks, plasma glucose (g) and NT-proBNP (h) determined after 6 weeks and after study end, respectively. Data are mean \pm SEM, ${ }^{* * *} p<0.005,{ }^{*} p<0.05$ versus placebo. UPCR, urinary protein/creatinine ratio; UGCR, urinary glucose/creatinine ratio; SEM, standard error.

(For figure see next page.) 


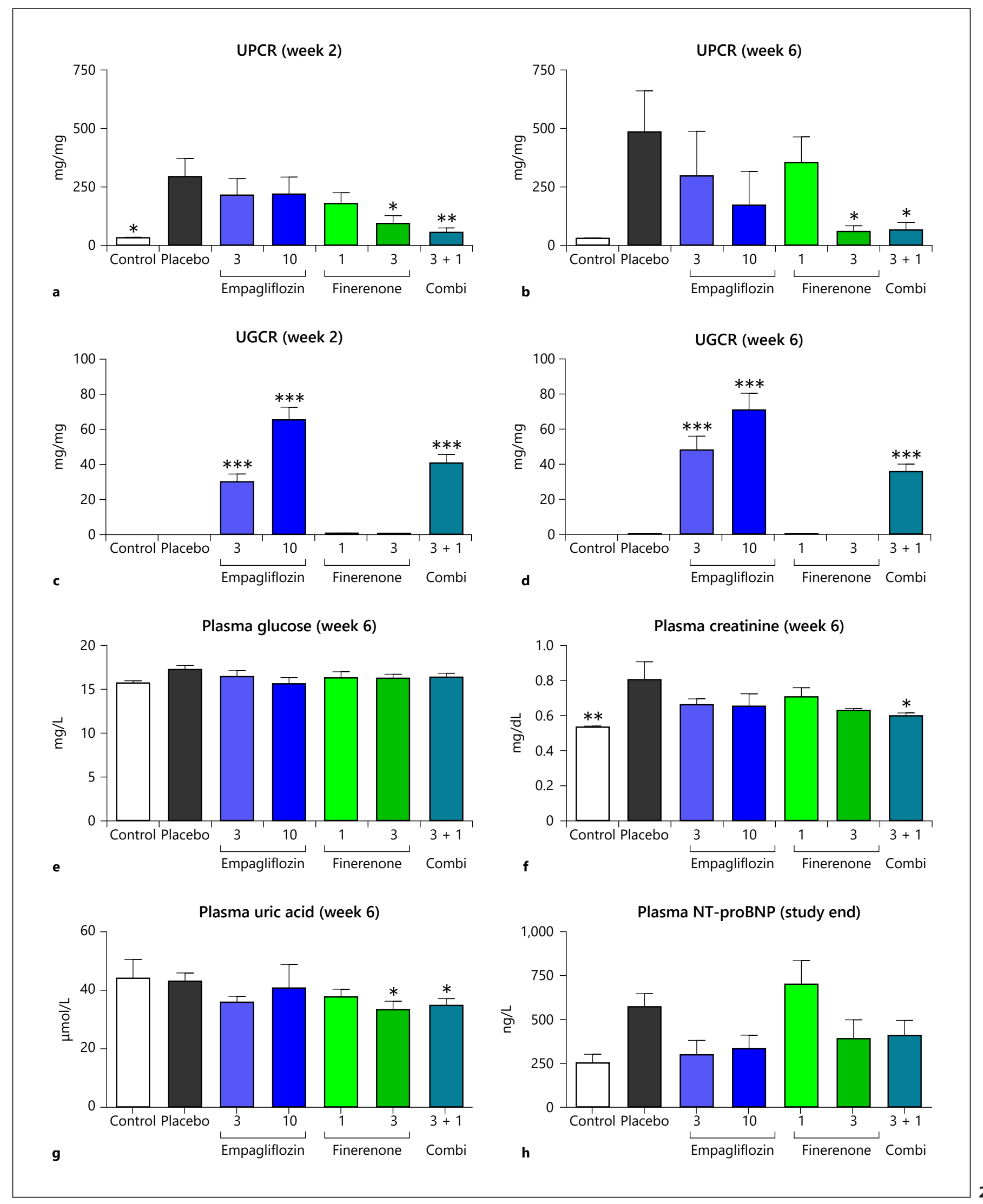


Table 1. SBP determined by the tail-cuff before the start of the treatment (week 0 ) and during the study in weeks 1,3 , and 5

\begin{tabular}{|c|c|c|c|c|c|c|c|}
\hline 0 & $113.0 \pm 2.5^{* * *}$ & $141.9 \pm 3.6$ & $153.9 \pm 4.4$ & $146.8 \pm 5.2$ & $155.7 \pm 3.8$ & $148.1 \pm 5.0$ & $152.4 \pm 3.2$ \\
\hline 1 & $134.7 \pm 4.3$ & $153.5 \pm 5.4$ & $169.1 \pm 7.1$ & $178.8 \pm 5.3^{* *}$ & $178.1 \pm 6.1^{*}$ & $170.8 \pm 5.0$ & $162.4 \pm 5.3$ \\
\hline 3 & $127.3 \pm 2.9^{* * *}$ & $216.0 \pm 7.1$ & $207.4 \pm 7.7$ & $199.0 \pm 10.5$ & $199.1 \pm 7.9$ & $175.6 \pm 7.0^{* *}$ & $172.3 \pm 7.4^{* * *}$ \\
\hline 5 & $128.8 \pm 4.5^{* * *}$ & $202.0 \pm 6.8$ & $199.4 \pm 10.4$ & $188.4 \pm 8.7$ & $170.0 \pm 9.0^{*}$ & $164.4 \pm 4.7^{* *}$ & $173.1 \pm 7.7^{*}$ \\
\hline
\end{tabular}

Data are mean \pm SEM. SBP, systolic blood pressure; SEM, standard error. ${ }^{*} p<0.05 .{ }^{* *} p<0.01 .{ }^{* * *} p<0.005$, versus placebo.

Blood Pressure Determination

Systolic blood pressure (SBP) was measured by the tail-cuff method (Noninvasive Blood Pressure Monitoring System, 9002-series; TSE Systems, Bad Homburg, Germany) before the start of the treatment and during the study in weeks 1, 3, and 5, $24 \mathrm{~h}$ after the last gavage and after a previous adaptation to the cuff.

\section{Histological Analysis}

At necropsy, hearts and kidneys were removed and fixed in $10 \%$ neutral buffered formalin or Davidson's solution, respectively, and embedded in paraffin. Paraffin sections were prepared and stained with hematoxylin and eosin or Sirius Red and Fast Green. The slides were analyzed using a semiquantitative scoring, ranging from grade 1 to 4 (grade 1, minimal/very few; grade 2, slight/few/ small; grade 3, moderate; grade 4 , marked/many). The grading was applied for each of the predominant hypertension-induced cardiac and kidney lesions, such as vasculopathy, glomerulopathy, and tubular degeneration as well as cardiac and renal interstitial fibrosis by a pathologist. For each group, an average score was calculated (sum of all severity scores divided by the animal number).

\section{Gene Expression}

Total RNA from the kidney and heart apex was isolated using Trizol (Invitrogen, ThermoFischer Scientific). RNA was reversely transcribed into cDNA with the ImProm II Reverse Transcription System (Promega) according to the manufacturer's instructions. Specific primers and fluorescein (FAM) and tetramethylrhodamine (TAMRA) probes were designed (online suppl. Table 1; see www.karger.com/doi/10.1159/000516213 for all online suppl. material), and qPCR was performed with the qPCR Master Mix Plus (Eurogentech; Seraing, Belgium) on a 7900 HT Fast RealTime PCR System (Applied Biosystems, ThermoFischer Scientific). Ct values were determined by Applied Biosystems's SDS Software (version 2.4) and normalized to the housekeeping gene Rpl32.

\section{Statistics}

All results are expressed as means. Error bars are given as standard errors. Statistical analysis was performed using GraphPad Prism Software v8.02 (La Jolla, CA, USA). Survival benefit between the placebo and verum groups was analyzed by a logrank test for trend. For all other parameters, one-way ANOVA followed by Dunnett's multiple comparisons test was used to determine statistical differences versus the placebo group. A probability value of $p<0.05$ was considered as significantly different.

\section{Results}

\section{Mortality}

Placebo-treated rats demonstrated a 50\% survival rate over the course of 7 weeks, as shown in Figure 1. Drug treatment resulted in variable degrees of survival benefit, with a moderate protection by the $3 \mathrm{mg} / \mathrm{kg}$ empagliflozin dose and both finerenone dosages, while combination of low dosages provided the strongest survival benefit weeks (93\% with a $p$ value of 0.0064 , logrank test for trend).

\section{Effects on Urinary Parameters}

Monotherapies with finerenone, with empagliflozin, or with the combination of the respective low dosages did not have a significant influence on urinary volume excretion after 2 weeks and after 6 weeks (online suppl. Fig. 1a, b). Placebo-treated animals developed proteinuria already after 2 weeks, and the urinary protein/creatinine ratio (UPCR) in this group almost further doubled until week 6 . Proteinuria was significantly reduced by the higher dose of finerenone and by the low-dose combination group already after 2 weeks, as shown in Figure 2a. Monotherapies of finerenone $(-27 \%$ at $1 \mathrm{mg} / \mathrm{kg}, p=\mathrm{ns} ;-87 \%$ at $3 \mathrm{mg} / \mathrm{kg}, p<0.05)$ and empagliflozin $(-38 \%$ at $3 \mathrm{mg} /$ $\mathrm{kg}, \mathrm{ns} ;-64 \%$ at $10 \mathrm{mg} / \mathrm{kg}$, ns) dose-dependently reduced proteinuria after 6 weeks, as shown in Figure 2b. Lowdose combination of finerenone and empagliflozin revealed an efficacious proteinuria reduction $(-86 \%, p<$ 0.05 ), which was over-additive. Empagliflozin strongly and dose-dependently acted glycosuric, while finerenone had no effect on the urinary glucose-to-creatinine ratio and had no influence when coadministered on the empagliflozin-mediated glucose excretion, as shown in Figure $2 \mathrm{c}$ and $\mathrm{d}$.

\section{Effects on Plasma Parameters}

Next, we examined the effects of mono- and combination therapies on fasted plasma glucose, creatinine, and 


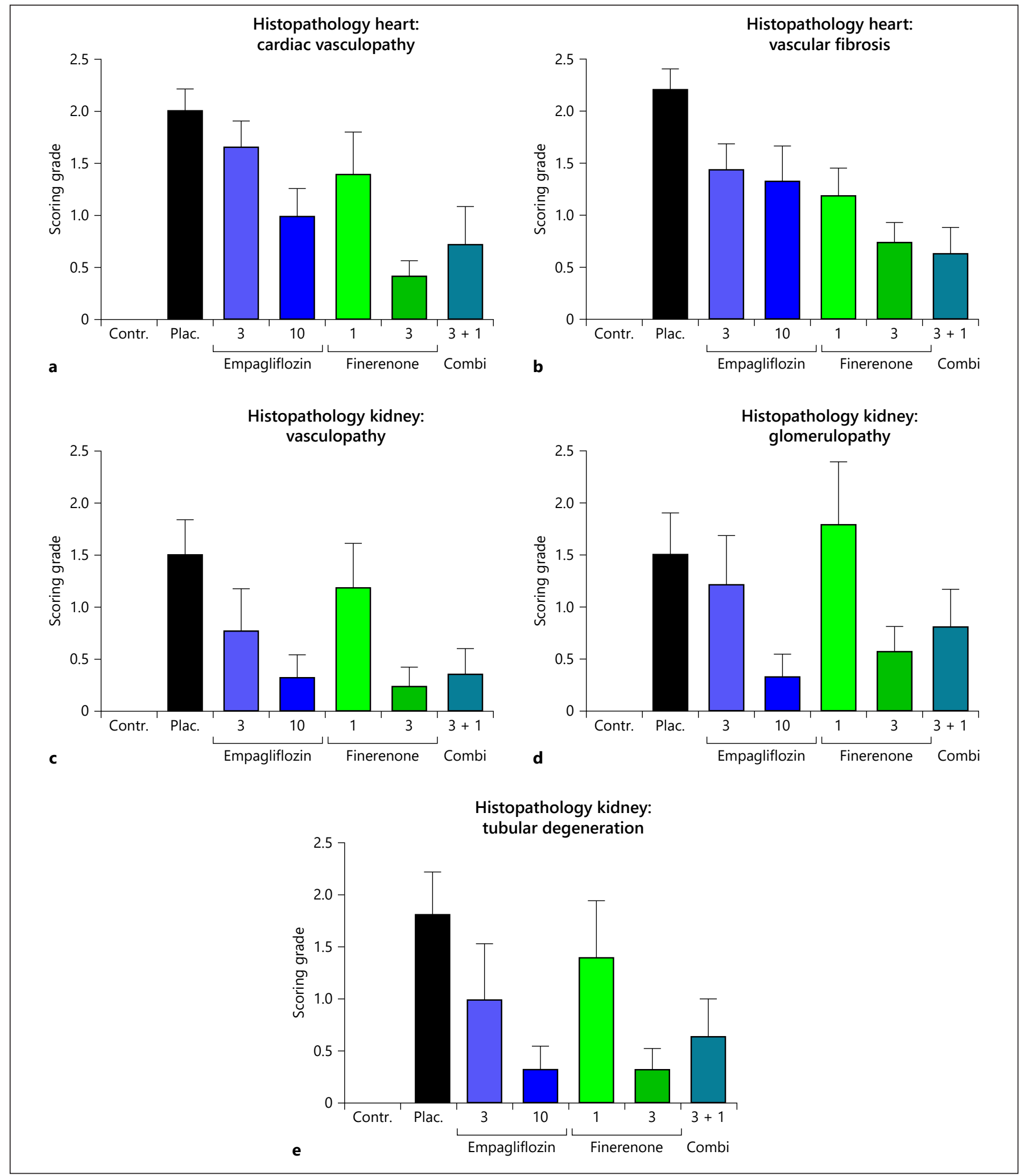

Fig. 3. Cardiorenal lesions as determined by semiquantitative histopathology: cardiac vasculopathy (a), cardiac vascular fibrosis (b), renal vasculopathy $(\mathbf{c})$, renal glomerulopathy $(\mathbf{d})$, and renal tubular degeneration (e). Data are mean \pm SEM. SEM, standard error. 
uric acid after 6 weeks. Figure 2e shows that both compounds, alone and in combination, had no significant influence on fasted plasma glucose in this nondiabetic animal model. There was a significant increase in plasma creatinine in placebo-treated animals. Although there was a trend of plasma creatinine reduction by all verum groups, only the low-dose combination group significantly reduced plasma creatinine after 6 weeks, as shown in Figure 2f. Treatment with $3 \mathrm{mg} / \mathrm{kg}$ finerenone and the low-dose combination significantly decreased plasma uric acid after 6 weeks, as shown in Figure 2g. At study end, plasma NT-proBNP was reduced by most verum groups with similar efficacy but without statistical significance, as shown in Figure $2 \mathrm{~h}$.

\section{Effects on Body Weight and SBP}

There was no significant difference in body weight gain among placebo, finerenone, empagliflozin, and combination groups until study end (online suppl. Table 2). Table 1 shows that mean SBP increased by ca. 74 $\mathrm{mm} \mathrm{Hg}$ in placebo-treated animals during the first 5 weeks of the study. Neither therapy with empagliflozin nor with low-dose finerenone decreased SBP significantly until the third week of the study. After 5 weeks of therapy, finerenone $(170.0 \mathrm{~mm} \mathrm{Hg}$ at $1 \mathrm{mg} / \mathrm{kg}$ and 164.4 at $3 \mathrm{mg} / \mathrm{kg}$ ) and the combination ( $173.1 \mathrm{~mm} \mathrm{Hg}$ ) significantly reduced SBP versus placebo $(202.00 \mathrm{~mm}$ $\mathrm{Hg}$ ), while therapy with empagliflozin had no significant effect on SBP. Blood pressure reduction was not different between the finerenone and combination groups.

\section{Effects on and Cardiac and Renal Organ Weights and} Histopathology

At study end, placebo-treated (mRen2)27 rats developed a significant increase in heart weight per body weight, but there was no statistical difference between the verum and the placebo groups (online suppl. Fig. 1c). There was also no statistical difference between the groups with respect to kidney weight per body weight (online suppl. Fig. 1d). Nevertheless, placebo-treated (mRen2)27 rats developed significant structural cardiac and renal damages. Histological analyses revealed myocardial degeneration including cardiac vasculopathy and

Fig. 4. Cardiac and renal fibrosis as determined by Sirius Red/Fast Green staining. Representative cardiac histology of control (a), placebo (b), and low-dose combination (c) animals. d Semiquantitative histopathology of cardiac fibrosis. Representative renal his- fibrosis as well as renal tubular degeneration, vasculoand glomerulopathy, and renal fibrosis. There was a dosedependent protection from cardiac vasculopathy, as shown in Figure 3a, and cardiac vascular fibrosis, as shown in Figure 3b, with both compounds, while lowdose combination therapy was more efficient than the respective monotherapy dosages on these cardiac histology parameters. Both compounds also yielded dose-dependent renal protection, as determined by histopathology shown in Figure $3 \mathrm{c}-\mathrm{e}$, for renal vasculopathy, glomerulopathy, and tubular degeneration with a comparable protection from renal lesions at higher dosages. However, the low-dose combination was generally more efficacious on renal histology parameters than the individual lowdose monotherapies.

\section{Effects on Cardiac and Renal Fibrosis}

Finally, we determined cardiac and renal fibrosis by Sirius Red/Fast Green staining. Placebo-treated (mRen2)27 rats developed significant cardiac and renal fibrosis over the course of the study compared with control rats as shown in Figure $4 \mathrm{a}, \mathrm{b}$, e and f, respectively. There was a dose-dependent protection from cardiac and renal fibrosis particularly by the low-dose combination therapy, as shown in Figure $4 c-g$. Semiquantitative scoring of cardiac and renal fibrosis revealed a comparable protection with the higher dosages of both compounds, while the low-dose combination was again more efficacious than the respective monotherapy dosages on cardiorenal fibrosis, as shown in Figure $4 \mathrm{~d}$ and $\mathrm{h}$.

\section{Effects on Gene Expression}

RNA-based expression analysis of biomarkers involved in renal damage, inflammation, fibrosis, and extracellular matrix turnover revealed that expression of the tubular damage marker kidney injury molecule-1 (KIM1), renal tissue inhibitor of metalloproteinases-1 (TIMP1 ), and renal collagen 1 a1 were significantly increased in placebo-treated animals in comparison to healthy control rats (online suppl. Fig. 2a-c). Expression of KIM-1 and TIMP-1 was significantly reduced by the low-dose combination group but not by the respective low-dose monotherapies (online suppl. Fig. 2a, b). Cardiac atrial natriuretic peptide expression was also significantly reduced by the low-dose combination and to some higher extend tology of control (e), placebo (f), and low-dose combination ( $(\mathbf{g})$ animals. $\mathbf{h}$ Semiquantitative histopathology of renal fibrosis. Data are mean \pm SEM. SEM, standard error. 

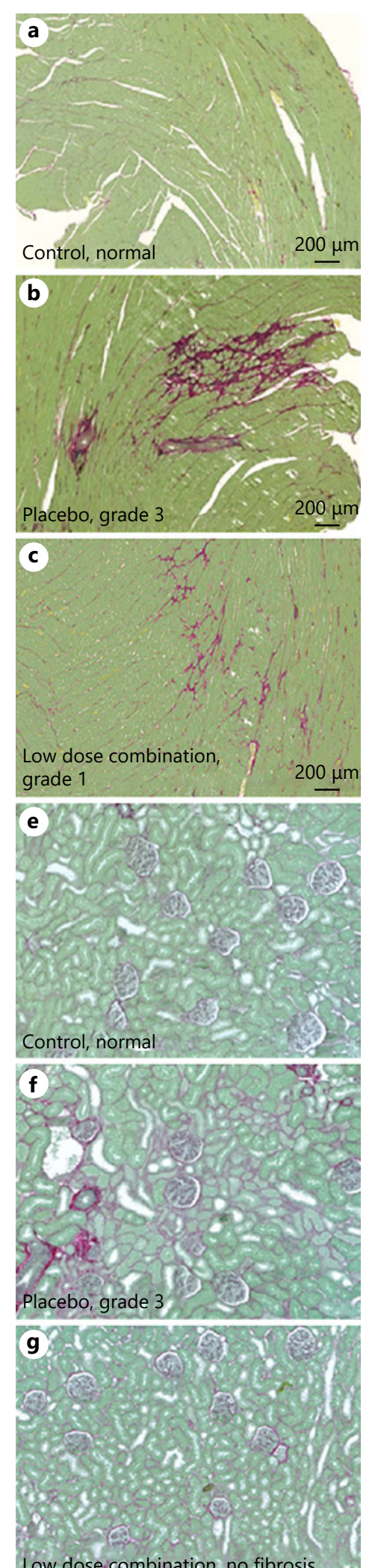

Low dose combination, no fibrosis
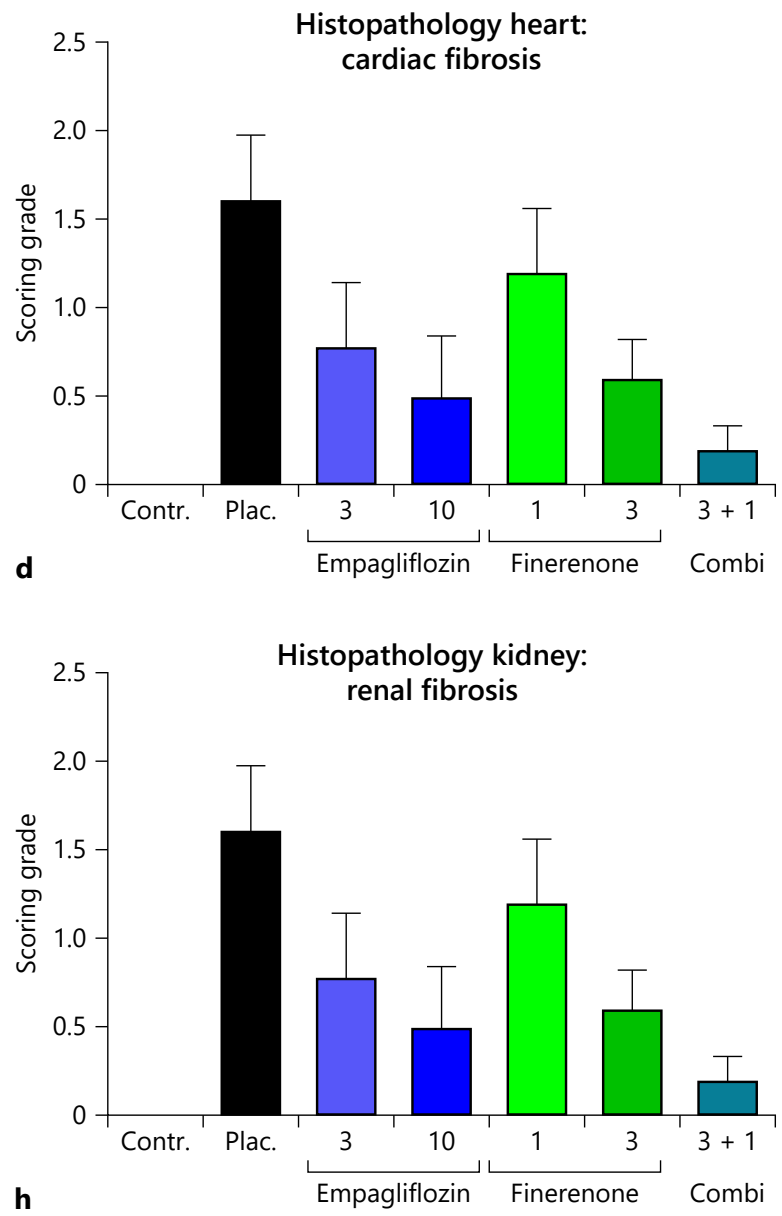

Preclinical Combination Therapy of Finerenone and Empagliflozin
Am J Nephrol 2021;52:642-652 DOI: $10.1159 / 000516213$ 
as the respective low-dose monotherapies (online suppl. Fig. 2d). Expression of cardiac osteopontin-1 and cardiac plasminogen activator inhibitor- 1 was not significantly increased between control- and placebo-treated animals (online suppl. Fig. 2e, f).

Taken together, combination of nonsteroidal MR blockade by finerenone with SGLT2i by empagliflozin at low dosages revealed efficacious reduction in proteinuria, plasma creatinine and uric acid, blood pressure, cardiac and renal lesions, and mortality.

\section{Discussion}

This is the first study describing beneficial effects of a combination of the nonsteroidal MRA finerenone with SGLT2i in preclinical nondiabetic cardiorenal disease. The major outcomes of the current preclinical study are that nonsteroidal MR antagonism by finerenone and SGLT2i by empagliflozin confer pronounced CV protection in hypertension-induced cardiorenal disease, and the combination of these 2 independent modes of action at low dosages revealed efficacious reduction in clinically meaningful parameters such as proteinuria, blood pressure, plasma creatinine, plasma uric acid, and mortality.

Diabetes and hypertension are the major causes of CKD. SGLT2 inhibitors and the nonsteroidal MRA finerenone have demonstrated clinical benefits in CKD patients with T2D $[11,21]$. In addition, SGLT2 inhibition by empagliflozin reduced outcomes in patients with HFrEF independent of the presence of T2D [5], and SGLT2i by dapagliflozin improved kidney and CV outcomes among patients with $\mathrm{CKD}$, regardless of the presence or absence of T2D [6]. Therefore, the benefit of SGLT2i seems not to be limited to a diabetic patient population, neither with HF nor with CKD. Similarly, antagonism of the MR provides benefit in preclinical models of $\mathrm{CV}$ diseases as well as in clinical studies with $\mathrm{HF}$ and $\mathrm{CKD}$ patients independent of the presence or absence of diabetes $[7,22,23]$, and therefore, both modes of action might be used in combination in broad cardiorenal patient populations.

We found a striking survival benefit from combination therapy in our hypertensive cardiorenal model which was accompanied by significant effects on renal function as deduced from plasma creatinine, plasma uric acid, and UPCR in the combination group. Therefore, these results in the first place rule out a theoretical functional antagonism between MR antagonism and SGLT2 $\mathrm{i}$ in hypertensive $\mathrm{CKD}$. Second, the results strongly argue for indepen- dent pharmacodynamic mechanisms which counteract the pathophysiological end-organ damage, and third, they even provide evidence for over-additive pharmacological efficacy, for example, as determined by UPCR despite an almost identical reduction in BP by low-dose finerenone and the low-dose combination group (see below). We also observed a dose-dependent reduction in histological lesions in both cardiac left ventricular as well as renal tissue with both compounds. Similarly, vascular, cardiac, and renal fibrosis were reduced by both modes of action with comparable efficacy at the higher dosages. Combination of respective low dosages was generally more efficacious on histology parameters than the individual low dosages. Surprisingly, we could only determine a nonsignificant trend for blood pressure lowering by empagliflozin, despite its very pronounced glycosuric activity. In contrast, blood pressure was dose-dependently and significantly lowered by finerenone and similarly by the low-dose combination treatment. These blood pressure data support the notion that the striking combined efficacy was derived from independent modes of action, with an overall more efficacious reduction in UPCR, end-organ damage, and mortality by the combined treatment.

Thus, the question arises which underlying molecular mechanisms are the basis for the observed efficacy in the combination group. Although the precise beneficial mode of action of SGLT2i in CKD and/or HF is not fully understood, it seems that a major component of these benefits is the counteracting activity of pathological hyper-reabsorption of sodium in the early proximal tubule via SGLT2, at least in the diabetic kidney [24]. This augmented reabsorption reduces the delivery of sodium to the macula densa, which, through tubulo-glomerular feedback, causes afferent arteriolar vasodilation and glomerular hyperfiltration. Attenuation of this proximal tubular hyper-reabsorption of sodium by SGLT2i causes afferent vasoconstriction, thereby lowering glomerular hyperfiltration and renal damage, and improves renal function in the long term. Other described pharmacodynamic effects of SGLT2 inhibitors include weight loss, systemic blood pressure reduction, ketone generation, and an increase in insulin sensitivity. Attenuating inflammation might be indirect long-term consequences of the primary metabolic/hemodynamic action $[25,26]$.

Under physiological conditions, MR also regulates sodium transport across the epithelia of the distal nephron by signaling cascades controlling membrane insertion of the epithelial sodium channel via transcriptional and according phosphorylation pathways [27]. However, patho- 
physiological conditions, such as those associated with an increased generation of reactive oxygen species, may cause a state of MR overactivation. MR overactivation in nonepithelial cells comprises conditions of chronically elevated aldosterone release, increased expression of MR, or aldosterone-independent MR activation, for example, by cortisol, another high-affinity ligand of MR, or via the small GTPase rac1, thereby generating a pro-inflammatory milieu leading to vascular, myocardial, and renal fibrosis, and eventually end-organ damage and failure [8, $9,28]$. Besides damage to tubular cells such as principal cells of the cortical collecting duct, several different cell types are involved in aldosterone-mediated renal injury including renal fibroblasts, mesangial cells, podocytes, and infiltrating macrophages [7]. The consequence of chronic MR overactivation in different glomerular, tubular, and vascular renal cell types can be inflammation, fibrosis, proteinuria, decreased kidney function, and eventually kidney failure $[8,26,29]$. Thus, based on extensive preclinical investigations and more recently even on clinical studies, MR blockade can be considered as an established anti-fibrotic mode of action for cardiorenal protection $[8,9,30]$.

Therefore, combination of the major underlying mechanisms, that is, metabolic/hemodynamic (SGLT2i) plus anti-inflammatory/anti-fibrotic (MR antagonism), based on the differential expression of the involved target proteins might yield chronic benefits throughout the end-organ heart and kidney which can exceed the efficacy provided by the respective monotherapies. More efficacious protection by this combination therapy was also supported by our preliminary mechanistic investigation at the RNA level since expression analysis of injury biomarkers such as cardiac atrial natriuretic peptide and renal KIM-1 as well as renal TIMP-1 as a marker for extracellular matrix turnover revealed also a more efficacious reduction by the combination therapy in comparison to the respective monotherapies. TIMP-1 concentrations were recently shown to be associated with new-onset $\mathrm{CKD}$ as well as with an increased risk of incident congestive HF in a community-based sample with almost 20 years of follow-up [31]. Interestingly, Packer and Kitzman [32] recently provided a mechanistic basis for the combined use of MRAs, SGLT2 inhibitors, and neprilysin inhibitors, in particular for obesity-related HF with preserved ejection fraction (HFpEF): in these patients, exacerbated sodium retention and plasma volume expansion promote cardiac and systemic inflammation and fibrosis. Therefore, inhibitors of MR overactivation, neprilysin, and SGLT2 should ameliorate the pathophysiological plasma volume expansion and pro-inflammatory and pro-fibrotic pathways.

Our study has several limitations including the unknown relevance of the chosen preclinical hypertensive (mRen2)27 rat model for human CKD patients. Another limitation is the chosen individual dosages of finerenone and empagliflozin in the single combination arm, while it is unclear if combination of higher dosages would have similar efficacy outcomes. Furthermore, the noninvasive tail cuff methodology for blood pressure monitoring is limited by difficulties with respect to movement artifact, the need for habituation to restraint, temperature, and other influences on the tail circulation [33]. Another important limitation is an increased rate in mortality over a relatively short study period which might lead to potential variations within groups due to sudden dropout.

In summary, combination of nonsteroidal MR antagonism by finerenone and SGLT2i by empagliflozin effectively reduces cardiac and renal lesions, proteinuria, and mortality in a nondiabetic hypertensive cardiorenal disease model, indicating a strong potential for combined clinical use in broad cardiorenal patient populations.

\section{Acknowledgements}

We would like to thank Petra Ammelung and Dorota Chodor for excellent technical assistance.

\section{Statement of Ethics}

All procedures conformed to European Community directives and national legislation (German law for the protection of animals) for the use of animals for scientific purposes and were approved by the competent regional authority.

\section{Conflict of Interest Statement}

All authors are employees of Bayer AG. The results presented in this paper have not been published previously in whole or part, except in abstract form at the Kidney Week Reimagined 2020 of the American Society of Nephrology (ASN).

\section{Author Contributions}

P.K. and P.S. provided the concept and design of research. P.K., E.H., A.F., and M.P. performed experiments and prepared figures. P.K., E.H., A.F., I.M., M.P., P.S., A.J., K.D., J.H., and F.E. analyzed data and interpreted results of experiments. P.K. and F.E. drafted the manuscript. P.K., E.H., A.F., I.M., M.P., P.S., A.J., K.D., J.H., and F.E. approved the final version of the manuscript. 


\section{References}

1 Ponikowski P, Voors AA, Anker SD, Bueno $\mathrm{H}$, Cleland JGF, Coats AJS, et al. ESC guidelines for the diagnosis and treatment of acute and chronic heart failure: the task force for the diagnosis and treatment of acute and chronic heart failure of the European Society of Cardiology (ESC) developed with the special contribution of the heart failure association (HFA) of the ESC. Eur Heart J. 2016 Jul 14; 37(27):2129-200.

2 Kidney Disease: Improving Global Outcomes (KDIGO) CKD Work Group. KDIGO 2012 clinical practice guideline for the evaluation and management of chronic kidney disease. Kidney Int Suppl. 2013:1-150.

3 Zinman B, Lachin JM, Inzucchi SE. Empagliflozin, cardiovascular outcomes, and mortality in type 2 diabetes. N Engl J Med. 2016 Mar 17;374(11):1094.

4 Wanner C, Inzucchi SE, Zinman B. Empagliflozin and progression of kidney disease in type 2 diabetes. N Engl J Med. 2016 Nov 3; 375(18):323-34.

5 Packer M, Anker SD, Butler J, Filippatos G, Pocock SJ, Carson P, et al. Cardiovascular and renal outcomes with empagliflozin in heart failure. N Engl J Med. 2020 Oct 8;383(15): 1413-24.

6 Heerspink HJL, Stefánsson BV, Correa-Rotter R, Chertow GM, Greene T, Hou FF, et al. Dapagliflozin in patients with chronic kidney disease. N Engl J Med. 2020 Oct;383(15): 1436-46.

7 Barrera-Chimal J, Girerd S, Jaisser F. Mineralocorticoid receptor antagonists and kidney diseases: pathophysiological basis. Kidney Int. 2019 Aug;96(2):302-19.

8 Kolkhof P, Jaisser F, Kim SY, Filippatos G, Nowack C, Pitt B. Steroidal and novel nonsteroidal mineralocorticoid receptor antagonists in heart failure and cardiorenal diseases: comparison at bench and bedside. Handb Exp Pharmacol. 2017;243:271-305

9 Agarwal R, Kolkhof P, Bakris G, Bauersachs J, Haller H, Wada T, et al. Steroidal and nonsteroidal mineralocorticoid receptor antagonists in cardiorenal medicine. Eur Heart $J$. 2021 Jan 7;42(2):152-61.

10 Bakris GL, Agarwal R, Chan JC, Cooper ME, Gansevoort RT, Haller H, et al. Effect of finerenone on albuminuria in patients with diabetic nephropathy: a randomized clinical trial. JAMA. 2015 Sep;314(9):884-94.

11 Bakris GL, Agarwal R, Anker SD, Pitt B, Ruilope LM, Rossing P, et al. Effect of finerenone on chronic kidney disease outcomes in type 2 diabetes. N Engl J Med. 2020 Dec; 383(23):2219-29.
12 Filippatos G, Anker SD, Agarwal R, Pitt B, Ruilope LM, Rossing P, et al. Finerenone and cardiovascular outcomes in patients with chronic kidney disease and type 2 diabetes. Circulation. 2021;143(6):540-52.

13 Mullins JJ, Peters J, Ganten D. Fulminant hypertension in transgenic rats harbouring the mouse Ren-2 gene. Nature. 1990 Apr 5; 344(6266):541-4.

14 Bachmann S, Peters J, Engler E, Ganten D, Mullins J. Transgenic rats carrying the mouse renin gene: morphological characterization of a low-renin hypertension model. Kidney Int. 1992 Jan;41(1):24-36.

15 Mullins LJ, Conway BR, Menzies RI, Denby L, Mullins JJ. Renal disease pathophysiology and treatment: contributions from the rat. Dis Model Mech. 2016 Dec 1;9(12):1419-33.

16 Mathar I, Kretschmer A, Hartmann E, Kolkhof P, Sandner P. Combination of soluble guanylate cyclase stimulation and mineralocorticoid receptor antagonism as new treatment option for heart failure with preserved ejection fraction $(\mathrm{HFpEF})$ : results from a preclinical study with vericiguat and finerenone. Circulation. 2017;136:A17778. (abstract, scientific session of the American Heart Association 2017)

17 Engler S, Paul M, Pinto YM. The TGR(mRen2)27 transgenic rat model of hypertension. Regul Pept. 1998 Oct;77(1-3):3-

18 Barfacker L, Kuhl A, Hillisch A, Grosser R, Figueroa-Perez S, Heckroth $\mathrm{H}$, et al. Discovery of BAY 94-8862: a nonsteroidal antagonist of the mineralocorticoid receptor for the treatment of cardiorenal diseases. ChemMedChem. 2012 Aug;7(8):1385-403.

19 Kolkhof P, Delbeck M, Kretschmer A, Steinke W, Hartmann E, Bärfacker L, et al. Finerenone, a novel selective nonsteroidal mineralocorticoid receptor antagonist protects from rat cardiorenal injury. J Cardiovasc Pharmacol. 2014 Jul;64(1):69-78.

20 Hansen HH, Jelsing J, Hansen CF, Hansen G, Vrang N, Mark M, et al. The sodium glucose cotransporter type 2 inhibitor empagliflozin preserves $\beta$-cell mass and restores glucose homeostasis in the male zucker diabetic fatty rat. J Pharmacol Exp Ther. 2014 Sep;350(3):65764.

21 Perkovic V, Jardine MJ, Neal B, Bompoint S, Heerspink HJL, Charytan DM, et al. Canagliflozin and renal outcomes in type 2 diabetes and nephropathy. N Engl J Med. 2019 Jun 13; 380(24):2295-306
22 Jaisser F, Farman N. Emerging roles of the mineralocorticoid receptor in pathology: toward new paradigms in clinical pharmacology. Pharmacol Rev. 2016 Jan;68(1):49-75.

23 Parviz Y, Iqbal J, Pitt B, Adlam D, Al-Mohammad A, Zannad F. Emerging cardiovascular indications of mineralocorticoid receptor antagonists. Trends Endocrinol Metab. 2015 Apr;26(4):201-11

24 Vallon V. The mechanisms and therapeutic potential of SGLT2 inhibitors in diabetes mellitus. Annu Rev Med. 2015;66:255-70.

25 Wanner C, Marx N. SGLT2 inhibitors: the future for treatment of type 2 diabetes mellitus and other chronic diseases. Diabetologia. 2018 Oct;61(10):2134-9.

26 Luft FC. Solute excretion, metabolism, and cardio-renoprotection via two distinct mechanisms revolutionize clinical outcomes. Acta Physiol. 2020 Nov 29:e13589.

27 Fuller PJ, Young MJ. Mechanisms of mineralocorticoid action. Hypertension. 2005 Dec; 46(6):1227-35.

28 Nagase M, Ayuzawa N, Kawarazaki W, Ishizawa $\mathrm{K}$, Ueda $\mathrm{K}$, Yoshida S, et al. Oxidative stress causes mineralocorticoid receptor activation in rat cardiomyocytes: role of small GTPase Rac1. Hypertension. 2012 Feb;59(2): 500-6.

29 Barrera-Chimal J, Jaisser F. Pathophysiologic mechanisms in diabetic kidney disease: a focus on current and future therapeutic targets. Diabetes Obes Metab. 2020 Apr;22(Suppl 1): 16-31.

30 Bertocchio JP, Warnock DG, Jaisser F. Mineralocorticoid receptor activation and blockade: an emerging paradigm in chronic kidney disease. Kidney Int. 2011 May;79(10):105160.

31 Lieb W, Song RJ, Xanthakis V, Vasan RS. Association of circulating tissue inhibitor of metalloproteinases- 1 and procollagen type III aminoterminal peptide levels with incident heart failure and chronic kidney disease. J Am Heart Assoc. 2019 Apr 2;8(7):e011426.

32 Packer M, Kitzman DW. Obesity-related heart failure with a preserved ejection fraction: the mechanistic rationale for combining inhibitors of aldosterone, neprilysin, and sodium-glucose cotransporter-2. JACC Heart Fail. 2018 Aug;6(8):633-9.

33 Jamieson MJ, Gonzales GM, Jackson TI, Koerth SM, Romano WF, Tan DX, et al. Evaluation of the IITC tail cuff blood pressure recorder in the rat against intraarterial pressure according to criteria for human devices. Am J Hypertens. 1997 Feb;10(2):209-16. 